\title{
Trigeminal Nociceptive Transmission in Migraineurs Predicts Migraine Attacks
}

\author{
Anne Stankewitz, David Aderjan, Falk Eippert, and Arne May \\ Department of Systems Neuroscience, University Medical Center Hamburg-Eppendorf, D-20246 Hamburg, Germany
}

\begin{abstract}
Several lines of evidence suggest a major role of the trigeminovascular system in the pathogenesis of migraine. Using functional magnetic resonance imaging (fMRI), we compared brain responses during trigeminal pain processing in migraine patients with those of healthy control subjects. The main finding is that the activity of the spinal trigeminal nuclei in response to nociceptive stimulation showed a cycling behavior over the migraine interval. Although interictal (i.e., outside of attack) migraine patients revealed lower activations in the spinal trigeminal nuclei compared with controls, preictal (i.e., shortly before attack) patients showed activity similar to controls, which demonstrates that the trigeminal activation level increases over the pain-free migraine interval. Remarkably, the distance to the next headache attack was predictable by the height of the signal intensities in the spinal nuclei. Migraine patients scanned during the acute spontaneous migraine attack showed significantly lower signal intensities in the trigeminal nuclei compared with controls, demonstrating activity levels similar to interictal patients. Additionally we found-for the first time using fMRI—that migraineurs showed a significant increase in activation of dorsal parts of the pons, previously coined "migraine generator." Unlike the dorsal pons activation usually linked to migraine attacks, the gradient-like activity following nociceptive stimulation in the spinal trigeminal neurons likely reflects a raise in susceptibility of the brain to generate the next attack, as these areas increase their activity long before headache starts. This oscillating behavior may be a key player in the generation of migraine headache, whereas attack-specific pons activations are most likely a secondary event.
\end{abstract}

\section{Introduction}

Migraine is a disabling, recurrent headache, which is broadly divided into migraine with or without aura and some rare genetic types. Migraine is characterized by moderate to severe, often unilateral, and pulsating headache attacks aggravated by physical activity and accompanied by vegetative symptoms such as nausea, vomiting, photophobia, and phonophobia (Headache Classification Committee of the International Headache Society, 2004). From a pathophysiological point of view, migraine is understood as a paroxysmal CNS dysfunction. Neuronal circuits involved in migraine are of intense research interest (Lambert and Zagami, 2009). Although the pathophysiology of migraine is not yet fully understood, it has been postulated that peripheral events such as the activation of the trigeminovascular system lead to vasodilatation and plasma extravasation of meningeal vessels are most likely only a secondary event, as the origin of migraine attacks lies in the CNS (Goadsby, 2009). There are several brain structures that have been discussed as playing a crucial role in migraine disease (Hargreaves and Shepheard, 1999; May, 2009a, 2009b). Migraineurs are characterized by an alteration of the interictal cortical excitability level, pointing to a deficit of filter

Received Aug. 27, 2010; revised Nov. 8, 2010; accepted Nov. 11, 2010.

This work was supported by grants from the German Research Foundation (MA 1862/2-3) and Federal Ministry of Education and Research (371 5701 and NeurolmageNord). This work was further supported by an unrestricted scientific grant from Almirall (to S.A.)

Correspondence should be addressed to Dr. Arne May, Department of Systems Neuroscience, University of Hamburg-Eppendorf, Martinistrasse 52, D-20246 Hamburg, Germany. E-mail: a.may@uke.uni-hamburg.de.

DOI:10.1523/JNEUROSCI.4496-10.2011

Copyright $\odot 2011$ the authors $\quad 0270-6474 / 11 / 311937-07 \$ 15.00 / 0$ mechanisms that is believed to protect individuals against sensory overload (Aurora et al., 2007; Coppola et al., 2007). During the headache attack, the function of brainstem nuclei involved in endogenous pain control and pain transmission is thought to be altered (Afridi et al., 2005; Lambert and Zagami, 2009). Consequently, a dysfunction of peripheral and central parts of the trigeminovascular system is of specific interest (Borsook et al., 2006; DaSilva et al., 2007). A cardinal feature of migraine is recurring headache manifesting in attacks. However, a crucial question is: which structure of the CNS acts as a catalyst? Many brainstem areas, including dorsolateral pons, basilar pons, pontine tegmentum, medial longitudinal fasciculus, periaqueductal gray (PAG), and central midbrain, have been previously discussed (Weiller et al., 1995; Bahra et al., 2001; Cao et al., 2002; Afridi et al., 2005; Denuelle et al., 2007) and it is by no means clear at what time point and for how long these structures show abnormal activity (May, 2009b).

Using behavioral intensity ratings as well as functional magnetic resonance imaging (fMRI)-data, we aimed to study whether the trigeminal pain perception and the neuronal activity in response to trigemino-nociceptive input differs between migraineurs and healthy controls and whether alterations in migraineurs are associated with the migraine cycle, i.e., forthcoming attacks. Previous studies reported ambiguous data; some of them revealed increased pain ratings (decreased pain thresholds) in migraine patients (GiersePlogmeier et al., 2009; Sand et al., 2010), whereas others did not find any difference (Moulton et al., 2008). Interestingly, it has been observed that the pain thresholds in migraineurs are 
lower in the pre-attack state than interictally (Sand et al., 2008).

We used an experimental stimulation approach implemented within an event-related fMRI pain paradigm. To avoid tactile sensation on the skin and because all the material used in the magnet room has to be nonferromagnetic, we used an olfactometer for administering a small amount of ammonia gas intranasally. We have previously validated this approach in a group of healthy subjects using event-related fMRI and standardized trigeminal nociceptive stimuli and have described robust activation of the trigeminal system (Stankewitz et al., 2009).

\section{Materials and Methods}

Subjects

We aimed to study 20 migraine patients during their interictal pain-free migraine interval at least $72 \mathrm{~h}$ before their next and $72 \mathrm{~h}$ after their last headache period and 20 healthy age- and gender-matched control subjects. Consequently, migraine patients were controlled for last and forthcoming attacks.

Fifty migraine patients and 23 control subjects underwent our experimental design until we had complete datasets of 20 migraineurs during their interictal period and 20 healthy control subjects.

The datasets of seven migraine patients and three healthy controls had to be excluded from the study because of predefined exclusion criteria regarding movement parameters during the experiment (see Image processing and statistical analysis-fMRI, below), low pain ratings (see Study design, below), or clinical features (i.e., more than six attacks per month, taking prophylactic migraine or analgesic medications). Ten patients experienced a migraine attack in the next $72 \mathrm{~h}$ after scanning and were therefore in the preictal phase and 13 patients were scanned during acute headache attacks. These patients were excluded from the original part of the study in which we aimed to explore interictal migraine patients only. However, datasets of these preictal and ictal patients were included in later post hoc analyses, which were prompted by results from the original analysis (see Image processing and statistical analysisfMRI, below).

In the original study, we focused on trigeminal pain processing in response to trigemino-nociceptive stimulation in interictal migraine patients ( $n=20 ; 15$ females) compared with healthy controls $(n=20 ; 15$ females). To clarify specific questions regarding the height of the trigeminal activity specifically in the last days before the beginning of acute headache attacks (preictal) as well as during acute head pain (ictal), we included data of the additionally scanned patients (preictal, $n=10$, 10 females; ictal, $n=13 ; 9$ females) post hoc. Ictal patients were additionally examined at a second time point outside attacks for within-subject comparisons.

Demographic characteristics, clinical features, and pain ratings of migraine patients and healthy controls are presented in Table 1. Migraine diagnosis was based on classification of the International Headache Society (Headache Classification Committee of the International Headache Society, 2004). None of the patients suffered from any other neurological or psychiatric disorders and neither migraineurs nor controls were taking medication, including preventative medication for migraine. Ictal patients $(n=13)$ were scanned during the first $6 \mathrm{~h}$ after their spontaneous headache attack started, and none of the patients treated their attack before scanning.

Healthy controls had no history of neurological or psychiatric disease, particularly no history of headache or other pain syndromes. Seven of the 24 healthy subjects who took part in our validation study (Stankewitz et al., 2009) were used for the present control group. All subjects gave their written, informed consent. The study was conducted according to the Declaration of Helsinki and approved by the Ethics Committee in Hamburg, Germany. Participants were informed in detail about the purpose of the study and about the stimulation procedure and they were also informed that they could withdraw from the experiment at any time. All of them were remunerated for participation.
Table 1. Demographic characteristics, clinical migraine features, and behavioral pain ratings of healthy controls and migraineurs (interictal, preictal, and ictal)

\begin{tabular}{|c|c|c|c|c|}
\hline & $\begin{array}{l}\text { Interictal } \\
\text { migraineurs }\end{array}$ & $\begin{array}{l}\text { Preictal } \\
\text { migraineurs }\end{array}$ & $\begin{array}{l}\text { Ictal } \\
\text { migraineurs }\end{array}$ & $\begin{array}{l}\text { Healthy } \\
\text { controls }\end{array}$ \\
\hline \multicolumn{5}{|l|}{ Demographics } \\
\hline Number & 20 & 10 & 13 & 20 \\
\hline Female/male & $15 / 5$ & $10 / 0$ & $9 / 4$ & $15 / 5$ \\
\hline Age range (years) & $20-39$ & $20-45$ & $20-46$ & $18-37$ \\
\hline \multicolumn{5}{|l|}{ Clinical characteristics } \\
\hline With/without aura & $7 / 13$ & $2 / 8$ & $1 / 9$ & \\
\hline Disease duration (years) & $12.7( \pm 8.1)$ & $10.1( \pm 4.2)$ & $12.2( \pm 5.1)$ & \\
\hline Attacks per month & $3(1-6)$ & $4(2-6)$ & $4(1-6)$ & \\
\hline \multicolumn{5}{|l|}{ Location of the pain } \\
\hline Right-sided predominantly & 2 & 4 & 2 & \\
\hline Left-sided predominantly & 4 & 2 & 3 & \\
\hline Changing sides & 14 & 4 & 8 & \\
\hline Last attack (days) & $4-21$ & $>3$ & & \\
\hline Next attacks (days) & $4-15$ & $1-3$ & $3 / 6$ & \\
\hline \multicolumn{5}{|l|}{ Attack severity* } \\
\hline (average and range) & & & $8(7-10)$ & \\
\hline \multicolumn{5}{|l|}{ Behavioral data } \\
\hline Averaged pain ratings & $6.7( \pm 2.3)$ & $6.2( \pm 3.4)$ & $6.5( \pm 2.3)$ & $6.5( \pm 2.4)$ \\
\hline
\end{tabular}

Mean values of the disease duration are presented with SD; mean values of pain ratings are presented with the SEM. *Attack severity was recorded on a numerical rating scale ranging from 0 (no pain) to 10 (highest imaginable pain).

\section{Study design}

The stimulation equipment and design used in the present study were described in detail previously (Stankewitz et al., 2009). Briefly, nociceptive stimulation of the trigeminal system was achieved using gaseous ammonia, which was applied to the receptive field innervated by the trigeminal nerve using an olfactometer. Intranasal application of ammonia stimulates the nasal mucosa and leads to an irritation of the first and second branch of the trigeminal nerve (V1 and V2), resulting shortlasting stinging or stabbing pain (Hummel and Kobal, 1992; McKeegan, 2004). Control conditions consisted of either an odor (rose), which mainly excites the olfactory nerve, or an odorless stimulus (air puffs). These substances were conveyed through a thin tube that was placed in subjects' right nostril.

At the beginning of each trial (Fig. 1), subjects underwent a reaction task (before they received stimuli). Each condition (i.e., ammonia, rose, air) was presented 15 times per session in a randomized order. The intensity of each stimulus was rated on a numerical rating scale (NRS), ranging from 0 (no pain) to 10 (highest imaginable pain). Before the fMRI experiment, subjects were trained with this paradigm outside the scanner. Only those subjects in which the given ammonia concentration evoked a clearly painful sensation (which was predefined as a 4 or above on the NRS) were included in the fMRI experiment. Subjects were instructed to breathe orally during the experiment to avoid fluctuations in stimulus concentrations due to respiratory airflow.

\section{Image acquisition}

MRI data were collected on a 3-tesla scanner (Siemens-Trio) using a 12-channel head coil. Functional images were acquired the following parameters: echo-planar imaging; forty-two axial slices; voxel size, $3 \times$ $3 \times 3 \mathrm{~mm}$; gap, $1 \mathrm{~mm}$; repetition time, $2620 \mathrm{~ms}$; echo time, $30 \mathrm{~ms}$; flip angle, $80^{\circ}$; field of view, $192 \times 192 \mathrm{~mm}$. As we were also interested in signal changes in brainstem structures, we paid particular attention to the alignment of the field of view. As a reference point for the lowest slice, we chose the caudal part of the cerebellum in each subject. Additionally, high-resolution T1-weighted structural images (voxel size, $1 \times 1 \times 1$ $\mathrm{mm}$ ) were acquired using a MPRAGE sequence.

\section{Analysis of behavioral data}

Behavioral data analysis was performed using SPSS (version 15.0; SPSS). The average pain ratings were calculated for all groups (interictal, preictal, and ictal migraineurs and control subjects) and an ANOVA was used for testing group differences between pain ratings. 


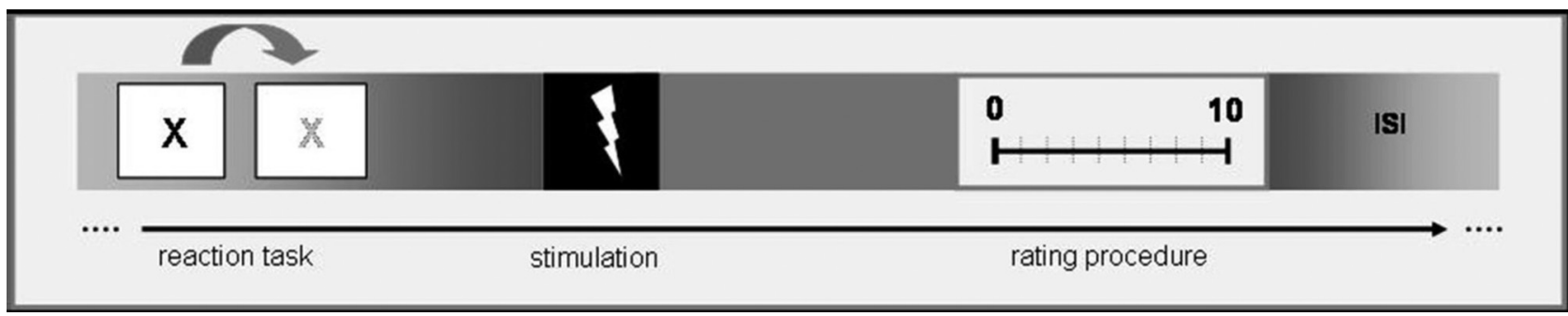

Figure 1. Experimental design. The stimulation paradigm consisted of three parts: reaction task, stimulation, and rating procedure. Before each of 45 trials (each stimulus was presented 15 times), a reaction time task was implemented. Subjects were instructed to press a button immediately after the fixation cross changed its color from red to yellow. After a jittered time delay, subjects underwent the trigeminal stimulation paradigm in which stimuli ( $2.5 \%$ ammonia, rose odor, air) were administered randomly in the right nostril. Following each stimulus, subjects rated the intensity on a numerical rating scale and a randomized interstimulus interval (ISI) followed.

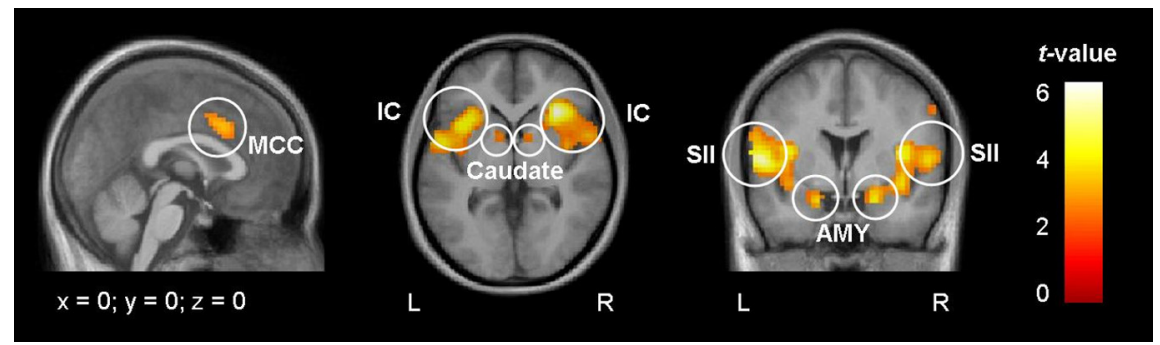

Figure 2. Activation pattern during nociceptive input. A conjunction analysis across both groups revealed shared activations of interictal migraineurs $(n=20)$ and healthy controls $(n=20)$ during trigeminal nociceptive stimulation of the right nostril. Increased BOLD responses were detected in several pain-processing areas including the insular cortex (IC), midcingulate cortex (MCC), secondary somatosensory cortex (SII), amygdala (AMY), and caudate nuclei (Caudate). Activation maps were plotted at a threshold of $p<0.001$ (uncorrected) and were overlaid onto the average structural image of healthy controls and interictal migraine patients. L, Left hemisphere; $R$, right hemisphere.

\section{Image processing and statistical analysis- $f M R I$}

fMRI data were preprocessed and statistically analyzed using SPM5 (Wellcome Department for Imaging Neuroscience, London, UK). Preprocessing included slice time correction, realignment (to the first volume), spatial normalization into the Montreal Neurological Institute (MNI) stereotactic space (MNI and International Consortium for Brain Mapping), and smoothing using a $10 \mathrm{~mm}^{3}$ full-width at half-maximum (FWHM) isotropic Gaussian kernel. In an additional control analysis (see Results), we used a smaller smoothing kernel (6 mm, FWHM). Motion parameters were checked for each subject. Subjects were only included in the analysis if their movements did not exceed $3 \mathrm{~mm}$ in translation and $3^{\circ}$ in rotation.

Statistical data analyses were performed using the general linear model. The following types of events were modeled as delta functions convolved with a canonical hemodynamic response function with its time derivative as implemented in SPM5: (1) ammonia (pain), (2) odour, (3) air puffs, (4) reaction time task (onset of fixation cross until button press after color change), (5) rating procedure (onset of the NRS until rating was finished), and (6) movement parameters (three translations and three rotations). The first-level design matrix of each subject thus consisted of the above mentioned 11 regressors. After model estimation, a linear contrast was defined to test for ammonia (pain) $>$ air puffs and a $t$ statistic was calculated at each voxel. As we focused our interest exclusively on trigeminal pain processing, we only report results of the pain contrast (ammonia $>$ air puffs). Data were analyzed for each subject individually in a first-level analysis and individual contrast images were then used a random-effects analysis that tests for group effects. Activations in the spinal trigeminal nuclei during nociceptive stimulation were of specific interest to us. It is thus important to note that slices down to $z=-52$ (MNI space) were available in all individuals, whereas the top slice scanned in all individual was $z=60$ (MNI space).

\section{Statistical tests performed at the group level}

Analyses of interictal migraine patients and matched controls. To test for similarities in pain-related activation across both groups (interictal mi- graineurs, $n=20$; matched control subjects, $n=20$ ), a conjunction analysis (Nichols et al., 2005) was performed. To test for differences between these two groups, a two-sample $t$ test (including nonsphericity correction for possible unequal variance of the error term in the two groups) was carried out; this latter analysis was also performed on the data that were smoothed with a $6 \mathrm{~mm}$ kernel.

In a further analysis, we tested whether the strength of blood oxygenation level-dependent (BOLD) responses in the spinal trigeminal nuclei during painful stimulation could predict the time until forthcoming migraine attacks. A regression analysis was calculated outside SPM (using SPSS) with the variables BOLD responses in the trigeminal nuclei and distance to the next headache attack (number of days). We therefore extracted the mean signal intensity (as obtained from the first-level contrast image) of the spinal trigeminal nuclei in all interictal patients $(n=20)$ by using a 4 -mm-radius sphere centered on coordinates from an earlier study by our group: $x=3, y=$ $-36, z=-45$ (peak activation during right-sided nasal nociceptive stimulation of the trigeminal nerve) (Stankewitz et al., 2009). A similar analysis was performed with the monthly attack frequency as the dependent variable.

Post hoc analysis comparing migraine patients (interictal, preictal, and ictal) and controls regarding BOLD responses in the spinal trigeminal nuclei. We further focused our interest exclusively on trigeminal activity in different groups of migraineurs (interictal, $n=20$; preictal, $n=10$; and ictal, $n=13$ patients $)$ and control subjects $(n=20)$. For each subject, we extracted the mean signal intensity of the spinal trigeminal nuclei (as described above) and then tested for group effects using an ANOVA in SPSS. Additionally, post hoc tests (Scheffé) were performed to test for specific group differences.

Post hoc analysis testing for attack-specific activation in migraineurs scanned during acute head pain. Finally, a paired $t$ test was performed to detect migraine attack-specific BOLD responses; data from ictal patients $(n=13)$ were compared with their own data examined outside migraine attacks.

In all above described analyses of fMRI data, we used a threshold of $p<$ 0.001 , uncorrected. Activations in the brainstem reflecting the spinal trigeminal nuclei were additionally corrected for multiple comparisons $(p<0.05)$, using a sphere with a $4 \mathrm{~mm}$ radius centered on the coordinates from our previous study $(x=3, y=-36, z=-45)$ (Stankewitz et al., 2009). For all other analyses (regression analyses and ANOVA), a threshold of $p<0.05$ was used.

\section{Results}

\section{Behavioral data}

The interictal $(n=20)$, preictal $(n=10)$, and ictal $(n=13)$ migraine patients and control subjects $(n=20)$ did not significantly differ in pain ratings. Mean values of pain ratings and 
Table 2. Results of the conjunction analysis that detects effects that are present in both groups (threshold, $p<0.001$, uncorrected)

\begin{tabular}{|c|c|c|c|c|c|c|}
\hline \multirow[b]{2}{*}{ Anatomical structures } & \multicolumn{2}{|c|}{ z values } & \multicolumn{2}{|c|}{$t$ values } & \multicolumn{2}{|l|}{ MNI coordinates } \\
\hline & $\mathrm{L}$ & $\mathrm{R}$ & $\mathrm{L}$ & $\mathrm{R}$ & L & $\mathrm{R}$ \\
\hline Secondary somatosensory cortex & 7.56 & 4.10 & 6.22 & 5.60 & $-54,-3,6$ & $54,-3,9$ \\
\hline Insula & 5.07 & 7.78 & 6.55 & 6.50 & $-39,15,3$ & $33,24,3$ \\
\hline Amygdala & 5.55 & 5.89 & 4.06 & 4.38 & $-18,0,-2$ & $21,3,-21$ \\
\hline Midcingulate cortex & 5.48 & 5.36 & 3.99 & 3.87 & $-6,15,36$ & $9,9,39$ \\
\hline Caudate nucleus & 4.45 & 4.51 & 3.05 & 3.10 & $-9,6,0$ & $12,6,3$ \\
\hline Supplementary motor area & & 4.48 & & 3.07 & & $9,9,60$ \\
\hline Thalamus & 4.43 & 3.80 & 3.03 & 2.49 & $-12,3,3$ & $9,-9,3$ \\
\hline Motor cortex & & 4.42 & & 3.02 & & $54,-3,51$ \\
\hline Cerebellum & 4.32 & & 2.94 & & $-15,-66,-51$ & \\
\hline Anterior cingulate cortex & 3.67 & & 2.39 & & $-3,36,15$ & \\
\hline
\end{tabular}

Coordinates are in $\mathrm{MNI}$ space.

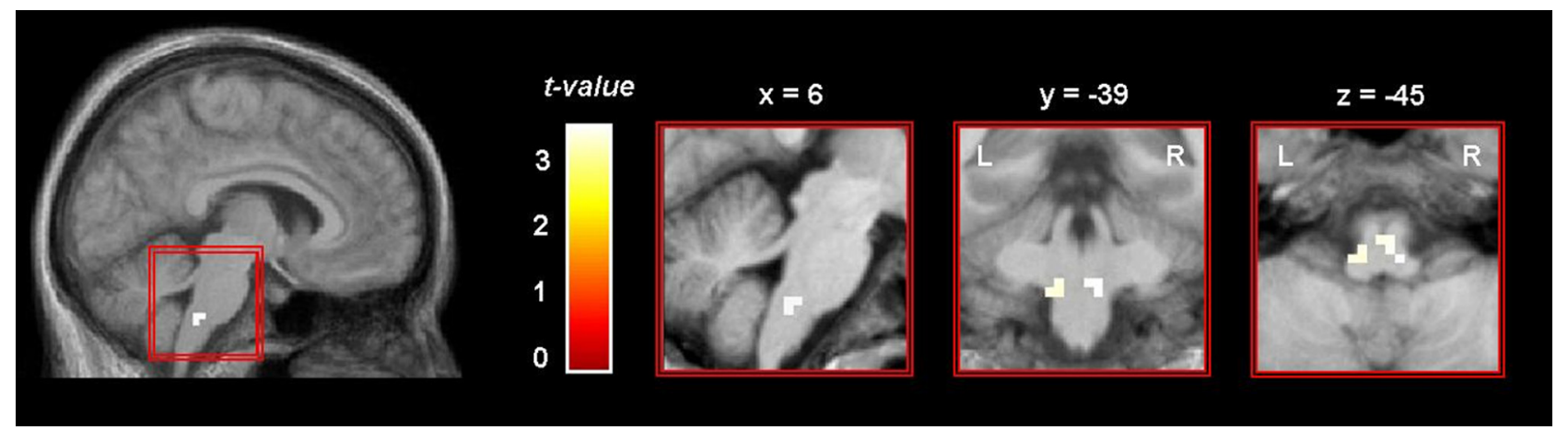

Figure 3. Comparison between interictal migraine patients and healthy controls. During trigemino-nociceptive stimulation of the right nostril, healthy controls ( $n=20$ ) showed significantly stronger bilateral activation than interictal migraineurs $(n=20)$ in a region of the brainstem corresponding to the spinal trigeminal nuclei. The activation is shown at a threshold of $p<0.001$ (uncorrected) and overlaid on the average structural image of healthy controls and interictal migraine patients. L, Left hemisphere; R, right hemisphere.

SEMs were as follows: interictal patients, $6.7 \pm 2.3$; preictal patients, $6.2 \pm 3.4$; ictal patients, $6.5 \pm 2.3$; controls, $6.5 \pm 2.4$ (Table 1).

\section{Imaging data}

Analyses of interictal migraine patients and matched controls Across both groups, increased BOLD signal changes during trigeminal nociceptive stimulation were detected in several cortical and subcortical brain areas, including the insula, midcingulate and anterior cingulate cortex, secondary somatosensory cortex, amygdala, cerebellum, caudate nuclei, and motor areas (Fig. 2; Table 2). A two-sample $t$ test revealed that controls showed significantly stronger activation in a brainstem area corresponding to the trigeminal nuclei (peak voxel: right, $x=6, y=-39, z=$ $-45, t_{(38)}=3.57$; left, $x=-9, y=-39, z=-45, t_{(38)}=3.36$; $p<0.001$, uncorrected) (Fig. 3). This finding also survived small volume correction $\left(t_{(38)}=3.43 ; p<0.01\right.$, corrected). The opposite contrast (patients greater than controls) did not reveal any differences. Because the smoothing kernel was $10 \mathrm{~mm}$ for this analysis (e.g., optimal for whole-brain analysis), and $10 \mathrm{~mm}$ may be too large for brainstem structures, we also performed an additional analysis on preprocessed data using a smaller kernel of 6 $\mathrm{mm}$ and confirmed the findings.

We additionally calculated correlation analyses between each of the six realign parameters and the pain regressor for each individual to make sure that the pain events were not influenced by head movements. Correlation coefficients did not reach values $>0.25$ and they did not significantly differ between groups.

A regression analysis revealed that the intensity of BOLD responses in the spinal trigeminal nuclei in interictal migraine patients $(n=20)$ significantly predicted the time until forthcoming

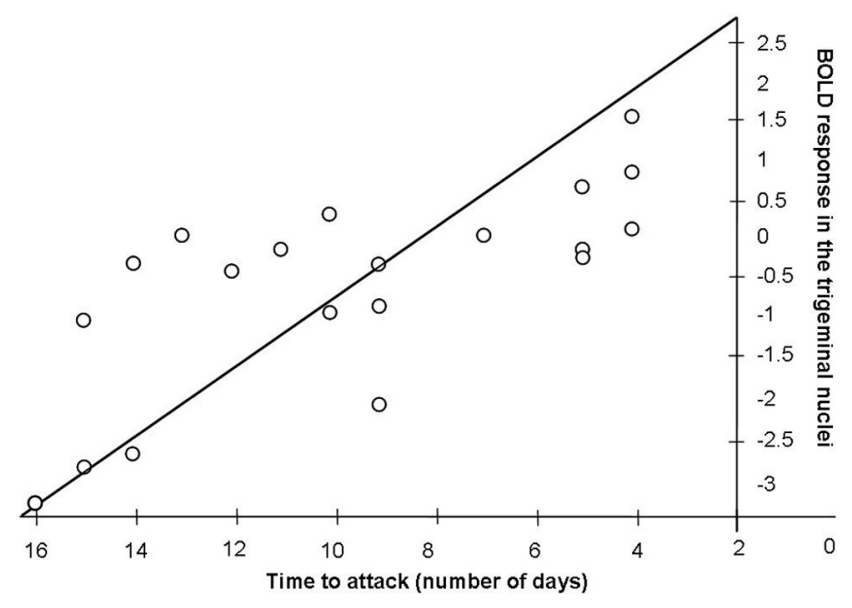

Figure 4. Relationship between BOLD responses and the time to the next attack. A regression analysis demonstrated that the intensity of the BOLD response in the spinal trigeminal nuclei (independent variable) during nociceptive stimulation predicts the time to the next attack (dependent variable; day 0 on the $x$ axis = headache attack) in the group of interictal migraine patients $(n=20)$. The diagonal line shows the regression.

attacks (number of days) — the stronger the activity within the trigeminal nuclei during painful stimulation, the closer migraineurs were to a migraine attack $\left(F_{(1,19)}=22.41 ; R^{2}=\right.$ $50.05 \%$; $p<0.001$ ) (Fig. 4). Even after excluding three extreme cases (the lowest data points on the very left side) (Fig. 4), the relationship remained significant $\left(F_{(1,19)}=5.30 ; R^{2}=26.13 \%\right.$; $p<0.03$ ). The second regression analysis (testing whether activity in the trigeminal nuclei predicts the monthly attack frequency) did not reveal a significant result $\left(F_{(1,19)}=0.38 ; R^{2}=\right.$ $2.10 \% ; p<0.886)$. 


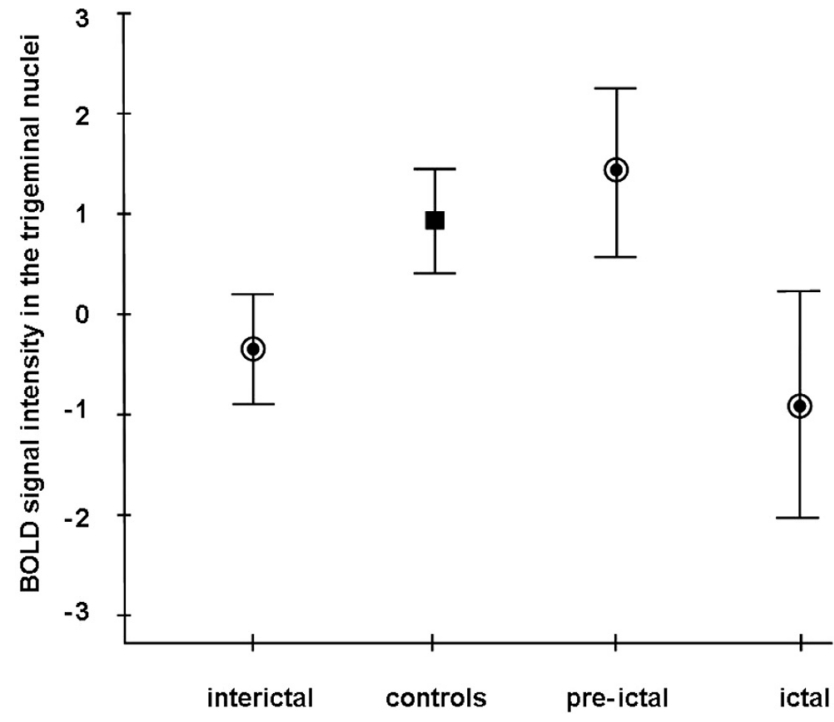

Figure 5. Group-specific BOLD responses in the trigeminal nuclei. The plot shows average trigeminal BOLD responses (mean and SEM) for interictal $(n=20)$, preictal $(n=10)$, and ictal ( $n=13$ ) migraine patients (circles) and healthy controls ( $n=20$; square). BOLD responses were obtained from a sphere of $4 \mathrm{~mm}$ radius placed around coordinates of the trigeminal nuclei $(x=3, y=-36, z=-45)$ that originated from a previous study (Stankewitz et al., 2009). attack compared with outside the attack $(x=0, y=-36, z=$ $-30 ; t_{(12)}=3.90 ; p<0.001$, uncorrected) (Fig. 6). The opposite contrast did not reveal any significant results.

\section{Discussion}

Comparing BOLD signal changes during trigeminal nociceptive input in migraine patients (exclusively within their interictal interval) and healthy controls, we found a robust activation pattern in cortical and subcortical areas well known from the pain literature (Peyron et al., 2000) that was shared by both groups. The sole difference between the two groups was detected in the lower brainstem; during trigemino-nociceptive stimulation, control subjects activated significantly stronger bilaterally in the lower pons, corresponding to the spinal trigeminal nuclei. This result was unexpected, as headache attacks depend on the activation of the complete trigeminal pain pathway, consisting of the trigeminal nerve fibers, the spinal trigeminal nuclei, the thalamus, and higher cortical areas (Borsook et al., 2004).

To delineate the functional relevance of this specific brainstem area in migraine patients, we tested for an association between the height of the activity in this region during the interictal period and the time until the next individual migraine attack. We observed that the height of BOLD responses in the trigeminal nuclei during trigeminal pain processing predicted the time until the next attack; i.e., the stronger the activation in the trigeminal nuclei, the nearer the attack. Importantly, BOLD response explained $>50 \%$ of the variance of this behavioral measure. These findings strongly suggest that the level of excitability of the spinal trigeminal nuclei is a fundamental part of the cyclical mechanisms generating the next migraine attack.

However, the finding that the BOLD response in the trigeminal nuclei in response to nociceptive stimulation shows a linear increase toward the next migraine attack contains no information about the absolute height of the activation level immediately before the headache attacks occur. Is the activity of spinal trigeminal Post hoc analysis comparing different groups of migraine patients with controls regarding $B O L D$ responses in the spinal trigeminal nuclei

A test for differences between the different groups of migraineurs (interictal, preictal, and ictal) and healthy controls regarding activation in the spinal trigeminal nuclei was highly significant $\left(F_{(3,62)}=6.13 ; p<0.001\right)$. Post hoc Scheffé tests showed that interictal migraine patients activated significantly less than controls $(p<0.034)$. Patients in the preictal state showed slightly more activation than controls (though far from significance) $(p<0.314)$ and a significantly enhanced activation compared with interictal patients $(p<0.012)$. Migraineurs during an acute headache attack showed significantly less activation than control subjects $(p<0.012)$ and significantly less activation than preictal migraineurs $(p<0.004)$. Mean values of the signal intensities and SEMs were as follows: interictal patients, $-0.346 \pm 0.280$; preictal patients, $1.404 \pm 0.422$; ictal patients, $-0.896 \pm 0.565$; controls, $0.913 \pm 0.254$ (Fig. 5).

Post hoc analysis testing for attack-specific activation in migraineurs scanned during acute head pain and outside of attacks

A paired $t$ test revealed that migraine patients showed significantly stronger activation in the rostral pons during an acute neurons in migraine patients shortly before and during an acute headache attack similar to controls or even higher? To address this point, we included data of preictal migraineurs, scanned between 12 and $48 \mathrm{~h}$ before their headache attack started, and ictal patients examined during acute head pain. These patients did not differ in any clinical features from interictal migraineurs; particularly, the perception of pain and the frequency of monthly attacks were similar in all groups.

We found that preictal migraine patients did not differ significantly from healthy controls but nevertheless showed a trend toward higher BOLD signal responses in the spinal trigeminal nuclei shortly before the attack. Interestingly, migraine patients scanned during the acute spontaneous migraine attack showed significantly lower signal intensities in the spinal trigeminal neurons compared with controls and preictal patients, demonstrating activity levels similar to interictal patients. In summary, the interictal BOLD signal in the trigeminal nuclei during nociceptive stimulation is generally lower than in controls, increases in height during the pain-free migraine cycle toward the migraine attack and is rapidly downregulated just before or immediately at the beginning of acute headache. It is noteworthy that the pain ratings did not differ between the groups and that only the pro- 
cessing of the trigeminal nociceptive input differentiates migraineurs from healthy controls.

It is somewhat surprising that differences between migraineurs and controls were observed exclusively at the level of the spinal trigeminal nuclei, but not in thalamic nuclei or in the somatosensory cortex, as information is relayed to these structures from the spinal trigeminal nucleus. Using fMRI, it is much more difficult to detect BOLD signal changes in the brainstem than in cortical regions because brainstem structures are very small and the physiological noise (of cardiac and respiratory nature) is much higher (Harvey et al., 2008). It is therefore unlikely that we have missed activations in higher areas while finding significant task-specific activations in the brainstem. One could argue that our finding is specifically driven by the trigeminal stimulus. Theoretically, subtle alterations may not be detectable using activation-based fMRI (as it relies on contrasting conditions but cannot make any inference regarding baseline differences) and that special techniques such as arterial spin labeling may reveal additional findings. A more likely explanation is that differences in the transmission of nociceptive input between migraineurs and controls in the spinal trigeminal nuclei are due to the inherent biological differences between these groups. It may well be that inhibitory and/or facilitatory mechanisms, perhaps even at different levels of the trigeminal-thalamic-cortical pathway, play a significant yet unrecognized role.

The present data cannot answer the question of whether the trigeminal pain system is dysfunctional in itself or whether other structures modulate its activity. It has to be said that spinal trigeminal nucleus is not a homogeneous structure and our analysis does not allow singling out specific subnuclei. Furthermore, the activation level of the spinal trigeminal nuclei may be significantly influenced by other parts of the brain. From a clinical view point, the hypothalamus would be the most likely modulator of the trigeminal pain system. Using positron emission tomography, a previous study reported increased cerebral blood flow bilaterally in the hypothalamus during an acute attack in migraine patients (Denuelle et al., 2007). However, the exact role of hypothalamic (dys)functions in migraine has not yet been entangled and should be addressed in further studies (Alstadhaug, 2009; Holland and Goadsby, 2009).

Other structures may operate as modulators of neuronal activity in the spinal trigeminal nuclei, namely the endogenous pain control system, such as the PAG or the raphe nuclei (Wang and Nakai, 1994). The PAG modulates nociception via a descending pathway that relays in the rostral ventromedial medulla (RVM) and terminates in the spinal cord. The RVM has distinct cell classes that directly inhibit or facilitate nociception (Fields, 2004; Kirouac et al., 2004). Further evidence for deficient inhibition comes from a study reporting hypofunctional structures in the midbrain in migraineurs (Moulton et al., 2008). Moreover, impaired iron homeostasis in the PAG (Welch et al., 2001; Kruit et al., 2009) and highly specific activation in the rostral pons (localization of the locus coreleus) during an acute migraine attack have been observed (Weiller et al., 1995; Afridi et al., 2005). In line with this latter finding, we also observed-for the first time using $\mathrm{fMRI}$ - that migraineurs showed a significant increase in activation of rostral parts of the pons during trigeminonociceptive stimulation. Increased BOLD signal changes in this brainstem region have not been reported in other acute or chronic pain states, or in other primary headache patients (such as cluster headache patients) (May, 2009b). This region has been therefore coined "migraine generator," and indeed in our study, it was present neither in pain-free migraineurs nor in control subjects. Given that we could not find this region in the preictal state and given the clinical progression of the migraine cycle, it is tempting to consider oscillating impulse generators in the limbic system, perhaps including the hypothalamus, which may have (indirect) modulating effects on the activation level of the trigeminal nuclei toward an attack, which is succeeded by a specific activation of the rostral parts of the pons in the actual headache attack. Either way, the spinal trigeminal nuclei are key structures with rising excitability toward a migraine attack, whereas the increased activation in the rostral pons, the so-called "migraine generator," occurs on a secondary level and only during the attack. Our finding highlights the importance of taking the time to the next attack into account when investigating migraine patients. Just as the migraine cycle spans several days and contains up to five phases (prodromi, aura, headache, resolution, and recovery) (Blau, 1992), the trigeminal activity in migraine patients is not constant but strongly variable (Ambrosini et al., 2003; Stankewitz and May, 2009). Averaged imaging data point to hyporeactive trigeminal neurons during trigemino-nociceptive stimulation in migraine patients compared with controls. However, individual data give us a more differentiated view as data differ from subject to subject depending on the time to the next migraine attack. Interpreting only averaged data in the attackfree interval will probably not allow general assumptions.

The present findings suggest that the trigeminal activity level during nociceptive stimulation significantly depends on the time point at which patients were scanned during their individual migraine interval. Further longitudinal studies are necessary in which migraine patients are examined repeatedly at different time points over their individual migraine cycle.

\section{References}

Afridi SK, Matharu MS, Lee L, Kaube H, Friston KJ, Frackowiak RS, Goadsby PJ (2005) A PET study exploring the laterality of brainstem activation in migraine using glyceryl trinitrate. Brain 128:932-939.

Alstadhaug KB (2009) Migraine and the hypothalamus. Cephalalgia 29:809-817.

Ambrosini A, de Noordhout AM, Sándor PS, Schoenen J (2003) Electrophysiological studies in migraine: a comprehensive review of their interest and limitations. Cephalalgia 23 [Suppl 1]:13-31.

Aurora SK, Barrodale PM, Tipton RL, Khodavirdi A (2007) Brainstem dysfunction in chronic migraine as evidenced by neurophysiological and positron emission tomography studies. Headache 47:996-1003.

Bahra A, Matharu MS, Buchel C, Frackowiak RS, Goadsby PJ (2001) Brainstem activation specific to migraine headache. Lancet 357:1016-1017.

Blau JN (1992) Migraine: theories of pathogenesis. Lancet 339:1202-1207.

Borsook D, Burstein R, Becerra L (2004) Functional imaging of the human trigeminal system: opportunities for new insights into pain processing in health and disease. J Neurobiol 61:107-125.

Borsook D, Burstein R, Moulton E, Becerra L (2006) Functional imaging of the trigeminal system: applications to migraine pathophysiology. Headache 46 [Suppl 1]:S32-S38.

Cao Y, Aurora SK, Nagesh V, Patel SC, Welch KM (2002) Functional MRIBOLD of brainstem structures during visually triggered migraine. Neurology 59:72-78.

Coppola G, Pierelli F, Schoenen J (2007) Is the cerebral cortex hyperexcitable or hyperresponsive in migraine? Cephalalgia 27:1427-1439.

DaSilva AF, Granziera C, Tuch DS, Snyder J, Vincent M, Hadjikhani N (2007) Interictal alterations of the trigeminal somatosensory pathway and periaqueductal gray matter in migraine. Neuroreport 18:301-305.

Denuelle M, Fabre N, Payoux P, Chollet F, Geraud G (2007) Hypothalamic activation in spontaneous migraine attacks. Headache 47:1418-1426.

Fields H (2004) State-dependent opioid control of pain. Nat Rev Neurosci 5:565-575.

Gierse-Plogmeier B, Colak-Ekici R, Wolowski A, Gralow I, Marziniak M, Evers S (2009) Differences in trigeminal and peripheral electrical pain perception in women with and without migraine. J Headache Pain 10:249-254. 
Goadsby PJ (2009) The vascular theory of migraine: a great story wrecked by the facts. Brain 132:6-7.

Hargreaves RJ, Shepheard SL (1999) Pathophysiology of migraine-new insights. Can J Neurol Sci 26 [Suppl 3]:S12-S19.

Harvey AK, Pattinson KTS, Brooks JCW, Mayhew SD, Jenkinson M, Wise RG (2008) Brainstem functional magnetic resonance imaging: disentangling signal from physiological noise. J Magn Reson Imaging 28:1337-1344.

Headache Classification Committee of the International Headache Society (2004) The international classification of headache disorders, 2nd edition. Cephalalgia 24[Suppl 1]:9-160.

Holland PR, Goadsby PJ (2009) Cluster headache, hypothalamus, and orexin. Curr Pain Headache Rep 13:147-154.

Hummel T, Kobal G (1992) Differences in human evoked potentials related to olfactory or trigeminal chemosensory activation. Electroencephalogr Clin Neurophysiol 84:84-89.

Kirouac GJ, Li S, Mabrouk G (2004) GABAergic projection from the ventral tegmental area and substantia nigra to the periaqueductal gray region and the dorsal raphe nucleus. J Comp Neurol 469:170-184.

Kruit MC, Launer LJ, Overbosch J, van Buchem MA, Ferrari MD (2009) Iron accumulation in deep brain nuclei in migraine: a population-based magnetic resonance imaging study. Cephalalgia 29:351-359.

Lambert GA, Zagami AS (2009) The mode of action of migraine triggers: a hypothesis. Headache 49:253-275.

May A (2009a) Morphing voxels: the hype around structural imaging of headache patients. Brain 132:1419-1425.

May A (2009b) New insights into headache: an update on functional and structural imaging findings. Nat Rev Neurol 5:199-209.

McKeegan DE (2004) Mechano-chemical nociceptors in the avian trigeminal muscosa. Brain Res Rev 46:146-154.
Moulton EA, Burstein R, Tully S, Hargreaves R, Becerra L, Borsook D (2008) Interictal dysfunction of a brainstem descending modulatory center in migraine patients. PloS One 3:e3799.

Nichols T, Brett M, Andersson J, Wager T, Poline JB (2005) Valid conjunction inference with the minimum statistic. Neuroimage 25:653-660.

Peyron R, Laurent B, Garcia-Larrea L (2000) Functional imaging of brain responses to pain: a review and meta-analysis (2000). Neurophysiol Clin 30:263-288.

Sand T, Zhitniy N, Nilsen KB, Helde G, Hagen K, Stovner LJ (2008) Thermal pain thresholds are decreased in the migraine preattack phase. Eur J Neurology 15:1199-1205.

Sand T, Nilsen KB, Hagen K, Stovner LJ (2010) Repeatability of cold pain and heat pain thresholds: The application of sensory testing in migraine research. Cephalalgia 30:904-909.

Stankewitz A, May A (2009) The phenomenon of changes in cortical excitability in migraine is not migraine-specific: a unifying thesis. Pain 145:14-17.

Stankewitz A, Voit HL, Bingel U, Peschke C, May A (2009) A new trigemino-nociceptive stimulation model for event-related fMRI. Cephalalgia 30:475-485.

Wang QP, Nakai Y (1994) The dorsal raphe: an important nucleus in pain modulation. Brain Res Bull 34:575-585.

Weiller C, May A, Limmroth V, Jüptner M, Kaube H, Schayck RV, Coenen HH, Diener HC (1995) Brainstem activation in spontaneous human migraine attacks. Nat Med 1:658-660.

Welch KM, Nagesh V, Aurora SK, Gelman N (2001) Periaqueductal gray matter dysfunction in migraine: cause or the burden of illness? Headache 41:629-637. 